\title{
ANALYZING $\eta^{\prime}$ PHOTOPRODUCTION DATA ON THE PROTON AT ENERGIES OF 1.5-2.3 GEV
}

\author{
K. NAKAYAMA \\ Department of Physics and Astronomy, \\ University of Georgia, Athens, GA 30602, USA \\ E-mail: nakayama@uga.edu \\ H. HABERZETTL \\ Center for Nuclear Studies, Department of Physics, \\ The George Washington University, Washington, DC 20052, USA \\ E-mail: helmut@gwu.edu
}

A combined analysis of the existing data on the reactions $\gamma p \rightarrow p \eta^{\prime}$ and $p p \rightarrow p p \eta^{\prime}$, based on a relativistic meson exchange model of hadronic interactions, is presented.

\section{Introduction}

One of the primary interests in investigating $\eta^{\prime}$ meson-production reactions is that they may be suited to extract information on nucleon resonances, $N^{*}$, in the less-explored higher $N^{*}$ mass region. Current knowledge of most of the nucleon resonances is mainly due to the study of $\pi N$ scattering and/or pion photoproduction off the nucleon. Since the $\eta^{\prime}$ meson is much heavier than a pion, $\eta^{\prime}$ meson-production processes near threshold necessarily sample a much higher resonance-mass region than the corresponding pion production processes. Therefore, they are well-suited for investigating high-mass resonances in low partial-wave states. Furthermore, these reactions provide opportunities to study those resonances that couple only weakly to pions, in particular, those referred to as "missing resonances".

Another special interest in $\eta^{\prime}$ photoproduction is the possibility to impose a more stringent constraint on its yet poorly known coupling strength to the nucleon. This has attracted much attention in connection with the so-called "nucleon-spin crisis" in polarized deep inelastic lepton scattering ${ }^{1}$ in that the $N N \eta^{\prime}$ coupling constant can be related to the quark contribution to the "spin" of the nucleon. ${ }^{2}$ Reaction processes where the $\eta^{\prime}$ meson 


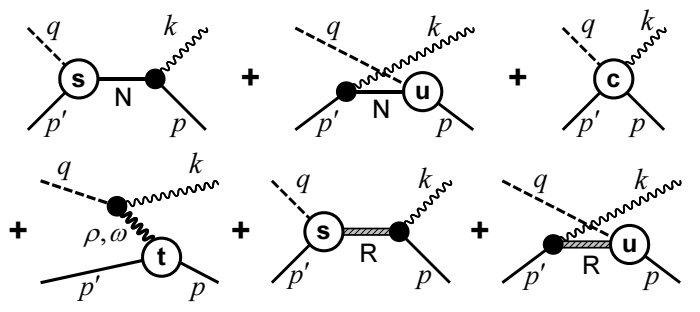

Figure 1. Diagrams contributing to $\gamma p \rightarrow \eta^{\prime} p$. The intermediate baryon states are denoted $\mathrm{N}$ for the nucleon, and $\mathrm{R}$ for the nucleon resonances. The total current is made gauge-invariant by an appropriate choice of the contact current depicted in the top-right diagram. The nucleonic current (nuc) referred to in the text corresponds to the top line of diagrams; the mesonic current (mec) and resonance current contributions correspond, respectively, to the leftmost diagram and the two diagrams on the right of the bottom line of diagrams.

is produced directly off a nucleon may offer a unique opportunity to extract this coupling constant.

The major purpose of the present contribution is to present the photoproduction part of a combined analysis of the $\gamma p \rightarrow p \eta^{\prime}$ and $p p \rightarrow p p \eta^{\prime}$ reactions within a relativistic meson-exchange model of hadronic interactions. The photoproduction reaction is described in the tree-level approximation, where a phenomenological contact term is introduced in order to guarantee the gauge-invariance of the full amplitude. ${ }^{3}$ The latter consists of nucleonic, mesonic and (nucleon) resonance currents as depicted in Fig. 1. The hadro-production reaction part of our analysis as well as further details can be found in Ref. 3 .

\section{Analysis of the SAPHIR data on $\gamma p \rightarrow p \eta^{\prime}$}

The objectives of analyzing the SAPHIR $\operatorname{data}^{8}$ on $\eta^{\prime}$ photoproduction are:

1) To shed light on the conflicting conclusions of the existing model calculations for these data. These contradictions are: (a) The origin of the shape of the observed angular distribution. Zhao ${ }^{4}$ has emphasized that this is due to the interference among the resonance currents, while Chiang et al. ${ }^{5}$ have concluded that it is due to the interference between the resonance and $t$-channel mesonic currents. Yet, Sibirtsev et al. ${ }^{6}$ have claimed that the mesonic current is responsible for the observed angular distribution. The latter authors use a $t$-dependent exponential form factor in their mesonic current. (b) $t$-channel meson exchange versus Regge trajectory. Chiang et 
al. ${ }^{5}$ have emphasized that the SAPHIR data can be described only if the Regge trajectory is used in the $t$-channel mesonic current, while other authors ${ }^{6,7}$ have used ordinary vector meson exchanges.

2) Can we constrain the $N N \eta^{\prime}$ coupling constant from the photoproduction reaction?

3) To which extent are we able to identify the nucleon resonances from the differential cross section data, i.e., can, for example, the mass of the resonance be pinned down from the existing cross-section data?

4) Provide inputs for the $N N \rightarrow N N \eta^{\prime}$ reaction.

Our results for the angular distribution at various energies are shown in Fig. 2, together with the SAPHIR data. ${ }^{8}$ First of all, the mesonic current is responsible for the measured forward peaked angular shape at higher energies. The $P_{11}$ resonance contribution is much larger than that due to the $S_{11}$ resonance. Also, note the interference effects among different currents; the $P_{11}$ resonance gives raise to a larger backward angle cross sections, while the total resonance current, $S_{11}$ plus $P_{11}$, yields a larger forward-angle cross sections. Adding the mesonic current leads to a further enhancement of the forward cross sections. We mention that the inclusion of the mesonic and $S_{11}$ resonance currents only is not sufficient to describe the strong angular dependence at lower energies. ${ }^{3}$ Also, the nucleonic current, through its interference with the mesonic and $S_{11}$ resonance currents, makes the angular distribution more pronounced, ${ }^{3}$ but not as pronounced as the addition of the $P_{11}$ resonance shown in Fig. 2a. It is clear, therefore, that the observed angular distribution is a result of the rather non-trivial interference among different currents.

Figure $2 \mathrm{~b}$ displays our results for various values of the $N N \eta^{\prime}$ coupling constant, $g_{N N \eta^{\prime}}$, in the nucleonic current. Here, we also include the mesonic and the $S_{11}$ and $P_{11}$ resonance currents. Note that the nucleonic current becomes pronounced at backward angles as the energy increases which is due to the $u$-channel diagram. We see that $g_{N N \eta^{\prime}}$ cannot be much larger than 3. Naively, we would expect that more accurate data at higher energies will enable us to reduce this upper limit. See, however, the analysis of the high-precision CLAS data in Sec. 3.

Next, we address the issue of the ordinary meson exchange versus Regge trajectory in the $t$-channel mesonic current. The mesonic current based on the ordinary vector-meson exchange contains an extra form factor at the electromagnetic vertex, while that based on the Regge trajectory contains 

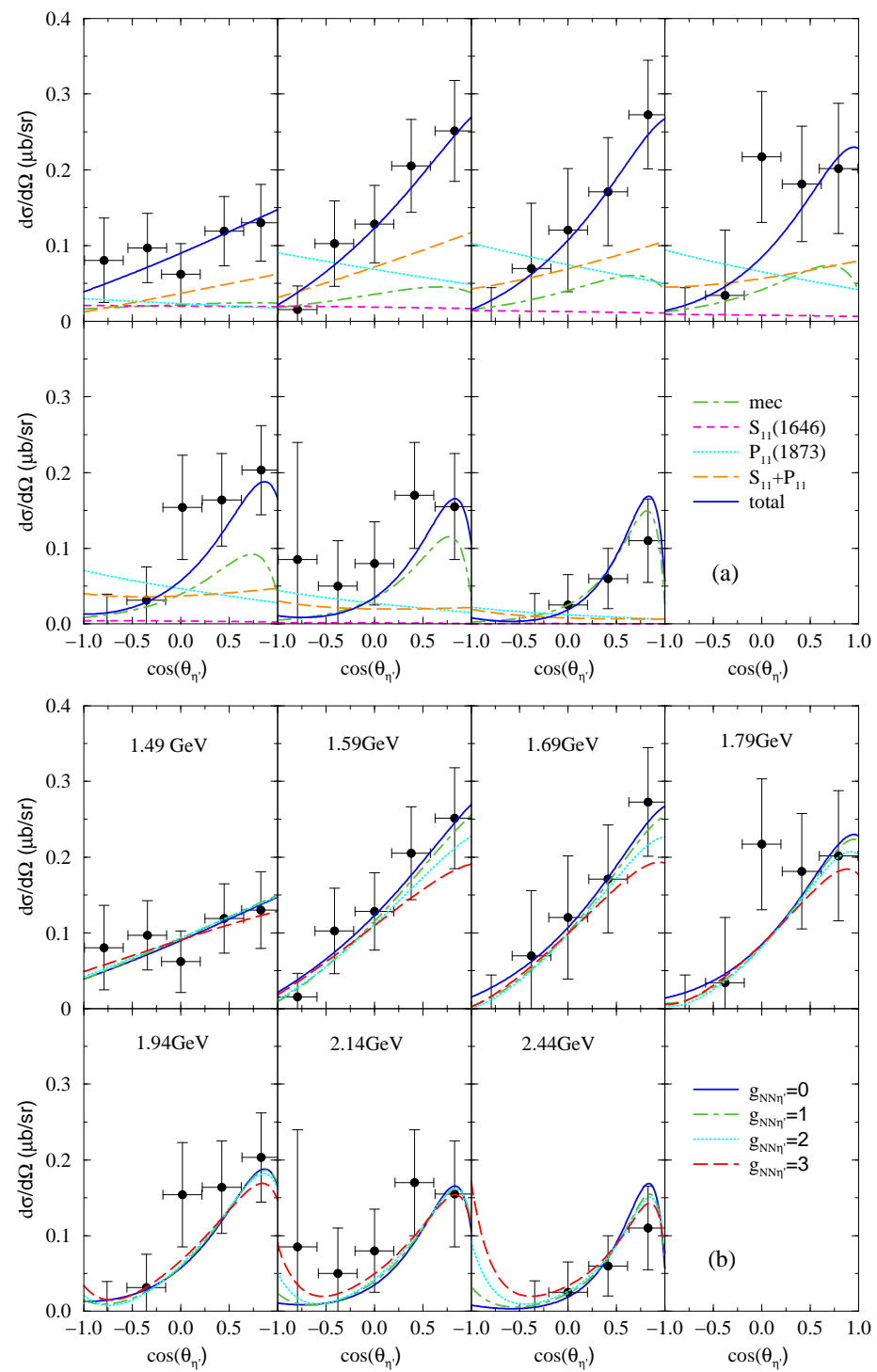

Figure 2. Differential cross section for $\gamma p \rightarrow p \eta^{\prime}$ according to the mechanisms shown in Fig. 1a. Panel (a) includes the meson-exchange current (mec), the $S_{11}$ and $P_{11}$ resonances. In (b), successively stronger (as indicated by the values of the $g_{N N \eta^{\prime}}$ coupling constant) nucleonic current (nuc) contributions are added to the results shown in panel (a). In each case, the model parameters are determined by best fits. The meaning of the corresponding lines is indicated in the panels. The data are from Ref. 8 . 
no such form factor. We verified that both models describe the data equally well, although there are some differences in detail. However, one important point to be noted here is that the resulting resonance parameters are quite different. This is quite disturbing, for it reveals a clear model dependence in the extracted resonance parameters. Further investigation of this important issue is required in future works.

We have also verified the sensitivity/insensitivity of the differential cross section data to the mass value of the nucleon resonance. In particular, we found that the results with the $S_{11}$ mass values which differ by about 100 $\mathrm{MeV}$ are hardly distinguishable from each other in the differential cross sections. This gives a rough idea about the uncertainty one should expect on the extracted resonance mass values based only on the differential cross section data.

\section{Analysis of the (preliminary) CLAS data on $\gamma p \rightarrow p \eta^{\prime}$ reaction}

Our model results for the (preliminary) CLAS data on $\eta^{\prime}$ photoproduction ${ }^{9}$ are shown in Fig. 3a. First of all, compared to the SAPHIR data ${ }^{8}$ analyzed in Sec. 2, the new CLAS data are much more accurate and, as such, may reveal features that were not seen in the analysis of the SAPHIR data. In Fig. 3a, different curves correspond to different sets of fit parameters which yield comparable $\chi^{2}$ values. Unlike the case of the SAPHIR data, here one requires not only the spin- $1 / 2$ resonances, but also the spin- $3 / 2$ resonances in order to reproduce the data. We found that the required spin- $1 / 2$ and $-3 / 2$ resonances are consistent with those quoted by the PDG. ${ }^{10}$

Although the different parameter sets yield practically the same differential cross sections (except for very forward and backward angles where no data exist), the corresponding dynamical content is very different from each other over the entire angular range. This shows, in particular, that cross sections alone are unable to fix the resonance parameters unambiguously, and that more exclusive observables, such as the beam and target asymmetries, are necessary in order to extract information on nucleon resonances.

Our predictions for the total cross section, as displayed in Fig. 3b, have been obtained by integrating the differential cross section results of Fig. 3a. A common feature present in all of these results is the bump structure around $W=2.09 \mathrm{GeV}$. If this is confirmed, the $D_{13}(2080)$ [and possibly $P_{11}(2100)$ ] resonance is likely to be responsible for the structure. The PDG ${ }^{10}$ quotes $D_{13}(2080)$ and $P_{13}(2100)$ as two and one star resonances, 

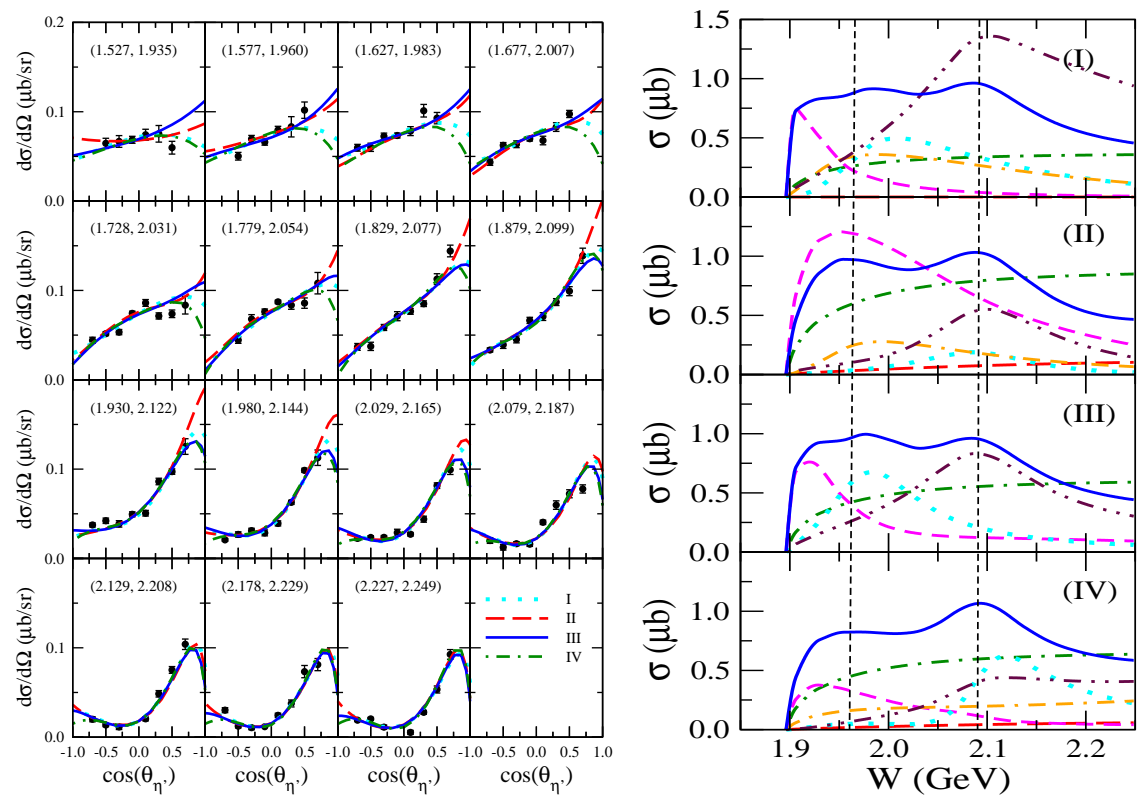

Figure 3. Left figure (a): Same as Fig. 2 for the CLAS data. ${ }^{9}$ The curves correspond to different fit results which yield comparable $\chi^{2}$ values. The numbers $\left(T_{\gamma}, W\right)$ in parentheses are the incident photon energy $T_{\gamma}$ and the corresponding $s$-channel energy $W=\sqrt{s}$, respectively, in GeV. Right figure (b): Total cross section for $\gamma p \rightarrow p \eta^{\prime}$ as a function of $W$. As indicated in the legend, the panels correspond to the fit results shown in the left figure. The overall total cross sections (solid lines) are broken down according to their dynamical contributions. The dash-dotted curves correspond to the mesonic current contribution; the dashed curves to the $S_{11}$ resonance current and the dotted curves to the $P_{11}$ resonance. The dot-double-dashed curves correspond to the $P_{13}$ resonance current while the dash-double-dotted curves show the $D_{13}$ resonance contribution. The nucleonic current contribution (long-dashed curves) are negligible and cannot be seen on the present scale. The two dashed vertical lines are placed to guide the eye through the two bump positions in all panels.

respectively. Another feature we see in Fig. 3b is the sharp rise of the total cross section near threshold which is caused by the $S_{11}$ resonance.

Contrary to the expectation in Sec. 2, the $N N \eta^{\prime}$ coupling constant cannot be determined even with the high-precision CLAS data. The reason is that even at higher energies, the resonance contribution may be large, especially that of the $D_{13}$ resonance. In fact, two of the results shown in Fig. 3b correspond to practically vanishing $g_{N N \eta^{\prime}}$. However, we are able to give a more stringent upper limit of $g_{N N \eta^{\prime}}<2$. In order to pin down further this coupling constant, one needs to either have more exclusive data 
than the cross section or go beyond the resonance energy region.

\section{Summary}

The study of $\eta^{\prime}$ production processes is still in its early stage of development. Our study reveals that in order to extract relevant physics, one needs more exclusive data than the cross sections. In addition, measurements of $\eta^{\prime}$ production using the neutron/deuteron target are also required. On the other hand, our theoretical model also needs to be improved; in particular, coupled channel effects should be investigated. In this connection, unfortunately, there is no realistic model for the $N \eta^{\prime}$ final state interaction available at present.

\section{Acknowledgments}

This work is partly supported by the Forschungszentrum Jülich, COSY Grant No. 41445282 (COSY-58).

\section{References}

1. J. Ashman et al., Phys. Lett. B206, 364 (1988).

2. G. M. Shore and G. Veneziano, Nucl. Phys. B381, 23 (1992).

3. K. Nakayama and H. Haberzettl, Phys. Rev. C69, 065212 (2004); nuclth/0507044.

4. Q. Zhao, Phys. Rev. C63, 035205 (2001).

5. W. T. Chiang et al., Phys. Rev. C68, 045202 (2004).

6. A. Sibirtsev, Ch. Elster, S. Krewald and J. Speth, AIP Conf. Proc. 717, 837 (2004); nucl-th/0303044.

7. B. Borasoy, Eur. Phys. J. A9, 95 (2000).

8. R. Plötzke et al., Phys. Lett. B444, 555 (1998).

9. M. Dugger and B. Ritchie, private communication.

10. S. Eidelman et al. (Particle Data Group), Phys. Lett. B592, 1 (2004). 